Table: Characteristics of G-Y, G-O, and G-OO

\begin{tabular}{lcccc}
\hline & G-Y & G-YO & G-OO & S.S. \\
\hline Cases & 207 & 142 & 227 & n.s. \\
female (\%) & 154 & 103 & 167 & n.s. \\
& $(74.4)$ & $(72.5)$ & $(73.6)$ & \\
age at onset & 48.1 & 61.8 & 71.4 & $p<0.01$ \\
age at baseline & 50.8 & 65.1 & 78.1 & p<0.01 \\
ACPA & 123.7 & 156.8 & 229.3 & n.s. \\
DAS28 at baseline & 2.7 & 3.2 & 3.1 & n.s. \\
Sharp/van de Heijde score at baseline & 30 & 46.6 & 65.6 & n.s. \\
HAQ at baseline & 0.292 & 0.478 & 0.84 & p<0.01 \\
PS-VAS at baselline & 37.3 & 41.5 & 43.4 & n.s. \\
DAS28<2.3 prevalence (\%) & 76.4 & 89.6 & 87.2 & n.s. \\
length untilDAS28<2.3 from baseline & 2.9 & 2.5 & 4 & n.s. \\
mean DAS28 at follor up & 1.6 & 1.4 & 1.5 & n.s. \\
mean DAS28 after DAS28 remission & 2.0 & 1.8 & 2.0 & n.s. \\
b-/ts-DMARD administration case and ratio (\%) & $41(19.8)$ & $29(20.6)$ & $41(18.0)$ & n.s. \\
mean MTX dosage & 8.6 & 8.6 & 7.4 & n.s. \\
GCS administration dosage and ratio (until & 2.96, & 2.41, & 2.71, & n.s. \\
DAS28 remission) & $24.2 \%$ & $38.0 \%$ & $42.6 \%$ & \\
GCS administration dosage and ratio (after & 5.68, & 4.58, & 2.14, & n.s. \\
DAS28 remission) & $19.3 \%$ & $21.1 \%$ & $26.4 \%$ & \\
\hline
\end{tabular}

Disclosure of Interests: None declared

DOI: 10.1136/annrheumdis-2019-eular.918

\section{AB0360 EVALUATION OF HEALTHCARE RESOURCE UTILISATION AND COSTS OF SJÖGREN'S SYNDROME: PATIENTS WITH RHEUMATOID ARTHRITIS TREATED WITH ABATACEPT OR ANTI-TNF DMARDS}

Evo Alemao ${ }^{1}$, Aarti Rao ${ }^{2}$, Joe Zhuo ${ }^{1}$, Chidananda Samal ${ }^{2}$, Robert Wong ${ }^{1}$, Paul Allison ${ }^{3} .{ }^{1}$ Bristol-Myers Squibb, Princeton, United States of America; ${ }^{2} \mathrm{Mu}$ Sigma, Bangalore, India; ${ }^{3}$ Statistical Horizons LLC, Ardmore, United States of America

Background: Sjögren's syndrome (SS) is considered an extra-articular manifestation of RA and is an autoantibody-mediated condition similar to RA. In an open-label, prospective, observational multicentre study, abatacept (ABA) was found to be effective for both RA and SS-related manifestations. ${ }^{1}$ However, there are limited data on the healthcare resource utilisation (HCRU) and cost in patients (pts) with RA with SS managed with ABA compared with those managed with anti-TNFs.

Objectives: To evaluate the HCRU and cost for pts with SS associated with RA treated with ABA or anti-TNF DMARDs.

Methods: Pts ( $\geq 18$ years) from the Truven MarketScan ${ }^{\mathrm{TM}}$ administrative claims database with incident RA $(\geq 2$ claims for RA using International Classification of Diseases [ICD]-9 or ICD-10 codes and $\geq 1$ claim for a conventional DMARD), incident SS ( $\geq 1$ claim for SS using ICD-9 or ICD10 codes) and with a prescription for ABA or an anti-TNF on or after the first diagnosis of SS from Jan 2011 to Sep 2017 were included. Pts were divided into two mutually exclusive cohorts. Pts with prescriptions for both ABA and an anti-TNF were excluded. The index date was the date of ABA or anti-TNF prescription. All-cause HCRU (healthcare visits) and costs were captured during the 2-year enrolled period prior to the index date (baseline) and in the 2-year enrolled follow-up period or until the pt was taken off the index drug, whichever occurred earlier. The baseline and follow-up periods were divided into intervals of 6 months each. Total healthcare visits (inpatient, outpatient, emergency care, urgent care and pharmacy) and total healthcare costs associated with these visits were calculated for each interval. A fixed-effects model was used to compare the HCRU and costs for pts taking ABA vs anti-TNFs after controlling for baseline Charlson Comorbidity Index and other comorbidities of interest.

Table 1

Table 1. Baseline characteristics for pts in the ABA and anti-TNF cohorts

\begin{tabular}{|l|c|c|}
\hline & ABA pts $(\mathbf{n}=148)$ & Anti-TNF pts $(\mathbf{n}=1219)$ \\
\hline Age, years & $56.0(11.0)$ & $53.0(11.0)^{\mathbf{*}}$ \\
\hline Female, $\mathbf{n}(\%)$ & $136(91.9)$ & $1065(87.4)$ \\
\hline Disease duration, days & $336.0(427.5)$ & $285.0(375.2)$ \\
\hline CCl score & $2.3(1.6)$ & $1.8(1.2)^{*}$ \\
\hline
\end{tabular}

Mean (SD) reported, unless stated otherwise

"p $<0.05$ vs ABA pts

$A B A=$ abatacept; $C C l=C h a r l s o n$ Comorbidity Index; pt=patient

Results: Overall, 1367 pts met inclusion criteria, of which 148 (10.8\%) and $1219(89.2 \%)$ were treated with $A B A$ and anti-TNFs, respectively. ABA (vs
anti-TNF) pts were older and had a higher comorbidity index (Table 1). Overall HCRU decreased in both the ABA and anti-TNF groups (relative to pts not being treated with biologic DMARDs). The HCRU was lower in ABA pts vs anti-TNF pts (average visits 20.0 vs 20.8. Figure 1) in the adjusted analysis. The average adjusted total healthcare cost for ABA (vs anti-TNF) pts was lower in the four 6-month cycles evaluated (average cost $\$ 17,050$ vs $\$ 27,469$; Figure 1). The overall healthcare costs were primarily driven by all-cause pharmacy cost (data not shown).

Conclusion: Pts with RA with SS managed with abatacept had lower HCRU and costs relative to pts managed with anti-TNFs. Further analysis is warranted to understand the drivers of the differential economic burden among pts with RA with SS.

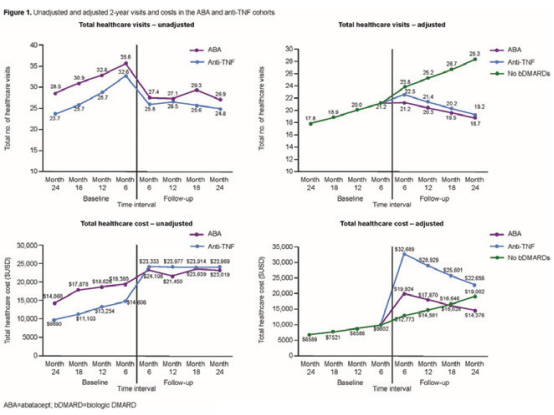

Figure 1

\section{REFERENCES}

[1] Tsuboi H, et al. Mod Rheumatol 2016;26:891-9.

Disclosure of Interests: Evo Alemao Shareholder of: Bristol-Myers Squibb, Employee of: Bristol-Myers Squibb, Aarti Rao Consultant for: Bristol-Myers Squibb, Joe Zhuo Shareholder of: Bristol-Myers Squibb, Employee of: Bristol-Myers Squibb, Chidananda Samal Consultant for: Bristol-Myers Squibb, Robert Wong Shareholder of: Bristol-Myers Squibb, Employee of: Bristol-Myers Squibb, Paul Allison: None declared

DOI: 10.1136/annrheumdis-2019-eular.2695

\section{AB0360B ANALYSIS OF SEQUENTIAL DEVELOPMENT OF PULMONARY LESIONS IN PATIENTS WITH RHEUMATOID ARTHRITIS}

Ayae Tanaka, Yuta Takamura, Tomoyuki Miyao, Ryutaro Yamazaki, Satoko Arai, Takayoshi Owada, Reika Maezawa, Masafumi Arima, Kazuhihiro Kurasawa. Dokkyo Medical University, Rheumatology, Tochigi, Japan

Background: Pulmonary involvement is critical for the management of RA. Pulmonary involvement shows various features in pathology and imaging such as interstitial pneumonia (ILD) and airway diseases (ADs). Importantly, pulmonary abnormalities coexist with other ones. We have previously reported that there were coexpstence patterns and ADs were shared abnormalities of patients with pulmonary lesions. However, it remains unknown through what pathways various pulmonary lesions develop.

Objectives: The purpose of this study was to determine the sequential development of pulmonary abnormalities in RA.

Methods: A retrospective cohort study. Subjects were consecutive 208 RA patients who were treated with bDMARDs as the first one from Feb.2004 to Sep. 2015 in our department and received HRCT scan before and during the therapy. Based on HR-CT imaging, pulmonary abnormalities were classified into 4 categories (ILD, nodular lesions, airway disease (AD) and others) and 20 lesions such as ground-glass opacity (GGO), reticular pattern, bronchiolitis and bronchiectasis. We recorded their existence and distribution and examined their changes. Cluster analysis was conducted according to new lesions at the second CT scans during the biological DMARDs, by Ward method. A checkerboard analysis with Chi-square test followed by residual analysis was conducted to examine the relation between pre-existing and newly emerg ing lesions. We compared the frequencies and pattern of newly emerging lesions in patients with or without pre-existing lesions.

Results: Subjects were 208 RA patients; M/F; 64/144, mean age; 59.2 years old, disease duration; 7.9 years, positive for RF in $84.1 \%$. bDMARDs used for the longest period were TNF inhibitors in $79.8 \%$ of the subjects, abatacept in $15.4 \%$ and tocilizmab in $4.8 \%$. Pulmonary 